\title{
GIS-based FRASTIC model for pollution vulnerability assessment of fractured-rock aquifer systems
}

\begin{abstract}
All groundwater is vulnerable to pollution, but the level of susceptibility depends on the spatial peculiarities of a region. Among the numerous vulnerability assessment methodologies, the geographical information system-based DRASTIC model is the most sophisticated and achieves reliable results even in complex areas. Fractured-rock aquifers are not only complex, but they are also potential sources of radioactive and other types of waste and are liable to high recharge rates. Hence, they can serve as a conduit for fluid (and potentially) contaminants. Therefore, in this study, a new parameter, 'fracture media' $(F)$, is fashioned to replace the weakest parameter (i.e. depth to water, $D$ ) in the standardized DRASTIC model to create a new model (FRASTIC) for the complex fractured aquifer system. The proposed model was tested on a case study area (Kano, Nigeria), and the FRASTIC minimum and maximum indices obtained were in the range of 63-170. The region was characterized as having very low (covering $0.16 \%$ of the total study area), low $(55.52 \%)$, medium $(42.53 \%)$, and high $(1.79 \%)$ vulnerability based on the standardized classification system. In addition, the modified FRASTIC model was further developed using the sensitivity analysis (SA) and recorded the highest vulnerable area coverage (32.86\%) within Kano. Thus, the modified FRASTIC model is appropriate for the complex nature of the study area because it contributes appreciably to defining the vulnerable zones. The single-parameter SA was employed to obtain effective weights for two modified models, which were then implemented to improve efficacy. Conventional nitrate validation indicated that there is a strong correlation between nitrate and vulnerability for the modified models. In addition, a new approach for identifying the relationship between the water quality and vulnerability indices was developed by this study and was proven to be an effective validation tool.
\end{abstract}

Keyword: Fracture; GIS; DRASTIC; FRASTIC; WQI; Nitrate; Kano 